\title{
Plasma boundary variability at Mars as observed by Mars Global Surveyor and Mars Express
}

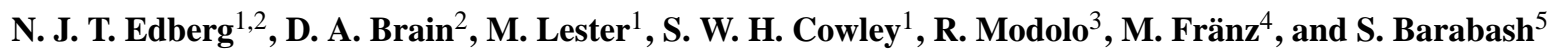 \\ ${ }^{1}$ Department of Physics \& Astronomy, University of Leicester, Leicester LE1 7RH, UK \\ ${ }^{2}$ Space Sciences Laboratory, University of California, Berkeley, USA \\ ${ }^{3}$ CETP-IPSL, 10-12 Avenue de l'Europe, 78140 Velizy, France \\ ${ }^{4}$ Max Planck Institute for Solar System Research, Katlenburg-Lindau, Germany \\ ${ }^{5}$ Swedish Institute of Space Physics, Kiruna, Sweden
}

Received: 15 June 2009 - Revised: 1 September 2009 - Accepted: 4 September 2009 - Published: 25 September 2009

\begin{abstract}
We have used Mars Express (MEX) and Mars Global Surveyor (MGS) simultaneous and non-simultaneous measurements to study the Martian plasma environment. In particular, we have derived quantitative expressions for the altitude of the terminator bow shock (BS) and magnetic pileup boundary (MPB) as functions of solar wind dynamic pressure, crustal magnetic fields and solar EUV flux. We have also studied the influence of the interplanetary magnetic field (IMF) direction. Through simultaneous two-spacecraft case studies we have shown that the dynamic pressure has a strong influence on the location and shape of these boundaries, which is also confirmed through a large statistical study. A higher dynamic pressure pushes the boundaries downward. The IMF direction has a weaker but still significant influence on both boundaries and causes them to move outward in the hemisphere of locally upward electric field. However, the MPB in the Southern Hemisphere is found to actually move inward when the electric field is directed locally upward. The crustal magnetic fields in the Southern Hemisphere have a strong influence on the MPB and cause it to move to higher altitudes over strong crustal magnetic fields. The influence of the crustal magnetic fields on the BS is more ambiguous since there are few crossings over the strongest crustal fields, but there appears to be at least a small trend of a higher BS for stronger crustal fields. An increased solar EUV flux has been found to cause the BS to move outward and the MPB to move inward.
\end{abstract}

Keywords. Interplanetary physics (Planetary bow shocks) Magnetospheric physics (Magnetosheath; Solar wind interactions with unmagnetized bodies)

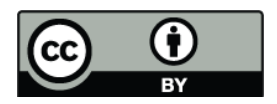

Correspondence to: N. J. T. Edberg (ne27@ion.le.ac.uk)

\section{Introduction}

Mars Global Surveyor (MGS) and Mars Express (MEX) form a unique pair of spacecraft that have made simultaneous and continuous measurements of the Martian plasma environment. MGS arrived at Mars in 1997 and performed measurements until late 2006, while MEX arrived in late 2003 and is still gathering data as of 2009 . Hence the two spacecraft obtained almost three years of simultaneous measurements. There have been many missions to Mars in the past equipped with plasma instruments which have individually studied Mars, but these two spacecraft are the only ones that have been in orbit at the same time and have hence enabled two-spacecraft studies. In this paper we will present results from case studies as well as statistical studies of simultaneous and non-simultaneous two-spacecraft measurements of the solar wind interaction with Mars. The aims of this study are to determine what effect the solar wind dynamic pressure, the interplanetary magnetic field (IMF) direction, the solar EUV flux and the crustal magnetic fields have on the location of the bow shock (BS) and magnetic pileup boundary (MPB) and to determine their relative importance.

Mars does not have a global intrinsic magnetic field, so that the solar wind interacts directly with the ionosphere. However, in the Southern Hemisphere where strong crustal fields are located the solar wind interaction can be significantly different (Acuña et al., 1998). The solar wind slows down to sub-sonic levels at the BS and the plasma becomes heated and turbulent forming the magnetosheath downstream of the BS. The magnetosheath stops at the MPB where the IMF starts to pile up and drape around the planet and the plasma population changes from solar wind plasma to planetary plasma (Nagy et al., 2004).

The shape and location of the Martian BS and MPB have been studied in the past by Slavin and Holzer (1981), Vignes et al. (2000), Trotignon et al. (2006) and Edberg et al.

Published by Copernicus Publications on behalf of the European Geosciences Union. 
(2008) among others. The coordinate system used in this paper to describe the location and shape of the boundaries is the Mars Solar Orbital (MSO) system, where the X-axis is directed toward the Sun, the z-axis is directed along the Mars orbital angular momentum vector and the y-axis completes the right-handed system. The shapes of both the BS and the MPB have been shown to be well represented by conic sections $r=L /(1+\epsilon \cos (\theta))$, where $r$ and $\theta$ are polar coordinates with origin at $X_{0}$ referenced to the MSO x-axis and $\epsilon$ and $L$ are the eccentricity and semi-latus rectum, respectively, of the conic section. At the subsolar point, where the boundaries are at their closest to the planet, the BS is on average located at an altitude of $0.58 R_{M}(\sim 2000 \mathrm{~km})$ and the MPB at an altitude of $0.33 R_{M}(\sim 1100 \mathrm{~km})$ (Edberg et al., 2008). The altitudes of the boundaries then increase on moving toward the nightside, such that on the day/night terminator the $\mathrm{BS}$ is on average at an altitude of $1.6 R_{M}(\sim 5400 \mathrm{~km})$ and the MPB at $0.45 R_{M}(\sim 1500 \mathrm{~km})$.

The variability of the altitude of the boundaries is very large and several factors are involved in determining their position. Factors that have been shown empirically to have an effect include the IMF direction (Vignes et al., 2002; Brain et al., 2005), the crustal magnetic fields (Crider et al., 2002; Dubinin et al., 2006; Fränz et al., 2006b; Edberg et al., 2008) and the solar wind dynamic pressure (Crider et al., 2003; Brain et al., 2005). Dubinin et al. (2008) also studied how the solar wind dynamic pressure balances the thermal pressure in the magnetosheath and the magnetic pressure inside of the MPB. When describing the shape of the boundaries as conic sections they are usually assumed to be rotationally symmetric around the $\mathrm{x}$-axis. However, due to the IMF direction and the crustal fields, the shapes can in fact be asymmetric and uneven. The crustal fields can, on average, cause the MPB to be $\sim 10 \%$ and the BS to be $\sim 20 \%$ farther out in the Southern Hemisphere than in the Northern Hemisphere (Edberg et al., 2008). The IMF direction can cause the BS to be $13 \%$ farther out in the hemisphere of locally upward electric field (Vignes et al., 2002). The studies mentioned above have all used single spacecraft data, except for Dubinin et al. (2006) who studied the MPB by using boundary crossings observed by MEX in combination with pressure and IMF direction observations from MGS. Also, Fedorov et al. (2006) studied the shape of the Martian wake and its relation to the IMF direction using MEX and MGS measurements.

\section{Instruments and orbits}

The two sets of instruments onboard MGS and MEX are complementary. MGS carries a magnetometer (MAG), which measures the vector magnetic field with a time resolution of up to $32 \mathrm{~Hz}$ and an electron reflectometer (ER), which measures suprathermal electrons in the energy range $10 \mathrm{eV}-$ $20 \mathrm{keV}$. ER has an energy resolution of $25 \%$, a time resolution of up to $2 \mathrm{~s}$ and a field of view of $14^{\circ} \times 360^{\circ}$, depending on telemetry rate and energy (Acuña et al., 1998; Mitchell et al., 2001). MGS does not carry an ion instrument.

MEX carries the ASPERA-3 instrument which includes an electron sensor (ELS) and an ion mass analyzer (IMA), but no magnetometer (Barabash et al., 2006). ELS is similar to ER and measures electrons in the energy range $10 \mathrm{eV}-20 \mathrm{keV}$. The maximum time resolution is $4 \mathrm{~s}$, the energy resolution is $8 \%$, and the field of view of $4^{\circ} \times 360^{\circ}$. IMA measures ions in the energy range $10 \mathrm{eV} / \mathrm{q}-30 \mathrm{keV} / \mathrm{q}$ and can resolve the masses of the main ion species (1, 2, 4, 8, 16 and $32 \mathrm{amu} / \mathrm{q})$. The full field of view (including electrostatic scanning) is $90^{\circ} \times 360^{\circ}$, and the time resolution of a full energy-elevation scan is $192 \mathrm{~s}$. The energy resolution is $7 \%$.

The orbits of the two spacecraft are very different. During the time of mission overlap (2004-2006) MGS was in a near-polar and near-circular $\sim 400 \mathrm{~km}$ altitude orbit fixed at 02:00-14:00 local time and with an orbital period of $2 \mathrm{~h}$. MEX was in a highly elliptical and precessing orbit with a periapsis at $\sim 270 \mathrm{~km}$ and an apoapsis at $\sim 10000 \mathrm{~km}$ with an orbital period of $6 \mathrm{~h}$.

\subsection{Measurements of and proxies for the dynamic pres- sure, IMF direction and solar EUV flux}

Crider et al. (2003) and Brain et al. (2005) have developed proxies for both the solar wind dynamic pressure and the IMF direction from MGS/MAG measurements. The proxies have been formulated in terms of measurements of the average magnetic field strength and draping azimuth (direction of the field) at $400 \mathrm{~km}$ altitude at latitudes between $50^{\circ} \mathrm{N}$ and $60^{\circ} \mathrm{N}$ where the crustal magnetic fields are weak. The field strength is assumed to balance, and therefore be a proxy for, the solar wind dynamic pressure. The magnetic field draping azimuth is assumed to be roughly the same as the clock angle of the IMF and is defined to be $0^{\circ}$ when the field is directed locally eastward and $90^{\circ}$ when locally northward. The pressure proxy is given by the magnetic field strength $B_{\text {proxy }}$ which can be converted to magnetic pressure $P_{B}=\frac{B_{\text {proxy }}^{2}}{2 \mu_{0}}$.

The solar EUV flux at Earth is determined through a proxy using F10.7 measurements of the radio flux at $2-200 \mathrm{~nm}$. The EUV flux at Earth can then be extrapolated to Mars, which has been used by Mitchell et al. (2001) and here we use the values developed in that study.

The solar wind (proton) velocity $v$ and density $n$ moments can be calculated from MEX/IMA measurements outside of the BS which is a more direct measurement of the solar wind dynamic pressure $P_{\mathrm{dyn}}=m_{p} n v^{2}$, where $m_{p}$ is the proton mass. Proton velocity and density moments are calculated from the nominal proton line in the IMA spectrogram for energies above $1 \mathrm{keV}$, and below that energy from a signal caused by protons scattered inside the IMA sensor. For details regarding the moment calculations, see Fränz et al. (2006a). The pressure determined from MEX that we will 

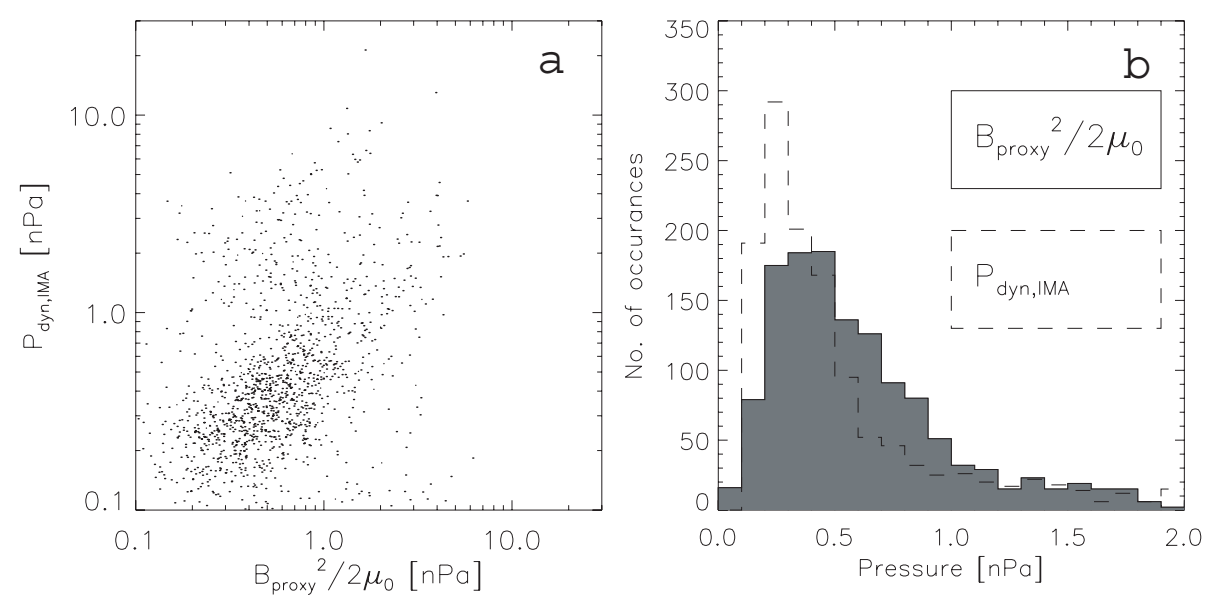

Fig. 1. (a) Measured solar wind dynamic pressure from MEX/IMA plotted vs. the MGS proxy for solar wind dynamic pressure and (b) distributions of the MGS proxy and the measured dynamic pressure from MEX/IMA.

use in this paper is calculated as a mean over $10 \mathrm{~min}$ of measurements exterior to a BS crossing.

The time resolution of both the MGS proxies are normally $2 \mathrm{~h}$ (one value per orbit) whereas the time resolution of the dynamic pressure measured by MEX is normally 2 samples per $6 \mathrm{~h}$ (one value inbound and one value outbound) and the time resolution of the EUV flux proxy is 1 to $2 \mathrm{~h}$.

In Fig. 1 we show a comparison between the MGS proxy for the solar wind dynamic pressure and the measured solar wind dynamic pressure from MEX. In panel (a) the MGS pressure proxy values are interpolated to the time of the MEX measured values and only data points during the overlapping mission time are included. There is a visible linear trend in the plot which indicates that the proxy is in reasonably good agreement with the measured values. However, there also seems to be a smaller population with high measured values from MEX while the MGS proxy shows low values, which could be explained by the fact that every high dynamic pressure event does not affect the plasma environment down to the altitude of MGS. The distributions of the measured values and the proxy values in panel (b) are similar but with slightly different mean values. The mean of the all the pressure proxy values is $0.74 \mathrm{nPa}$ while the mean of the measured MEX/IMA pressure values over all $10 \mathrm{~min}$ intervals exterior to BS crossings during the entire overlapping mission is $0.80 \mathrm{nPa}$. Both values have inherent uncertainties but are in reasonably good agreement.

\section{Mars Express and Mars Global Surveyor observations}

Since MEX is in a highly elliptical orbit with a low altitude periapsis it usually crosses both the MPB and the BS both inbound and outbound once every orbit. The BS is, how- ever, not always observed since the orbit of MEX is precessing and therefore MEX stays inside the BS during the entire orbit in some seasons. The BS is observed inbound as a sudden increase in fluxes of both electrons and ions in the ELS and IMA data sets. The MPB is observed inbound as a sudden decrease of magnetosheath electron and ion fluxes (examples of which will be presented in Figs. 3-5 below). The higher time resolution of ELS makes it easier to identify the exact location of the boundaries from that data set. The variability of the boundaries in combination with unfavorable orbit geometry makes it hard to identify the exact boundary location for many of the orbits and many crossings are therefore excluded. However, we have analyzed the entire set of MEX/ELS and MEX/IMA data from 2004 until 2009 and have identified 5014 MPB crossings and 3277 BS crossings. The number of crossings during the overlapping mission time between MGS and MEX (February 2004November 2006) decreases to 2500 and 1840, respectively, which is still twice as many as MGS observed from 1997 until 1999 when it was in an elliptic orbit similar to the orbit of MEX (Vignes et al., 2002; Trotignon et al., 2006; Edberg et al., 2008). It should be noted that we can only get values of the measured solar wind dynamic pressure when the BS is crossed, meaning that we do not know the upstream pressure for all of the 5014 MPB crossings.

During the overlapping mission time, MGS orbited Mars at $400 \mathrm{~km}$, well within the average MPB location and does not normally cross it. However, the variability of the magnetosheath is large and Brain et al. (2005) showed that for 5-20\% of the time, magnetosheath electrons are in fact observed at the altitude of MGS, such that the MPB had moved to altitudes below $400 \mathrm{~km}$. 


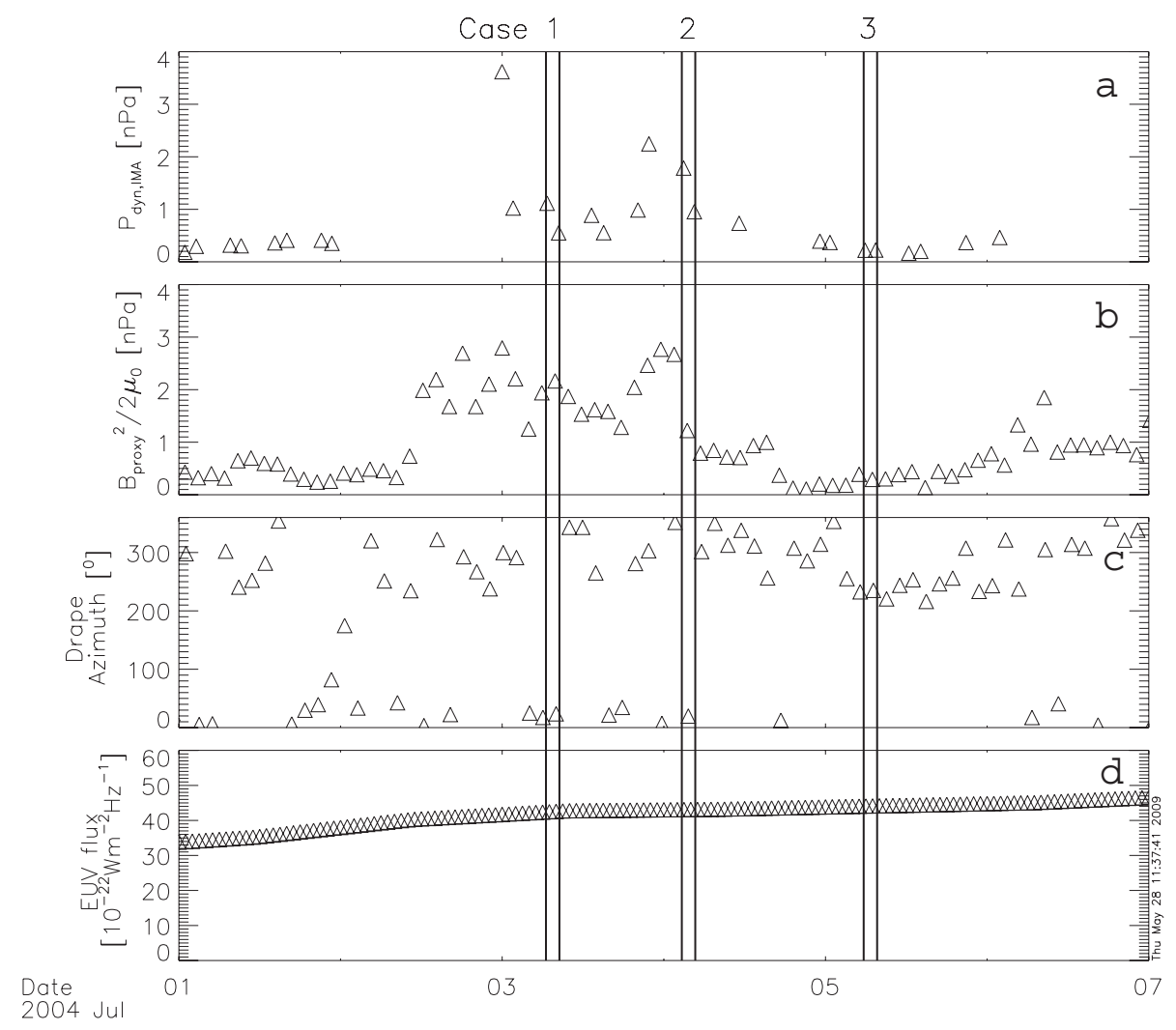

Fig. 2. Time series of (a) MEX measurements of solar wind dynamic pressure, (b) the MGS pressure proxy, (c) the MGS magnetic field draping direction and (d) solar EUV flux proxy. Three case studies of the variability of the MPB and BS are performed in Figs. 3-5 during the intervals indicated by the black vertical lines.

\section{Case studies with simultaneous measurements}

Using two-spacecraft simultaneous measurements we will show three case studies of how the solar wind dynamic pressure affects the position and shape of the Martian BS and MPB. Later, in Sect. 5, we will present results from statistical studies of how various factors influence the boundaries.

For the case studies, we have chosen an interval when the solar wind dynamic pressure starts at low values of $\sim 0.3 \mathrm{nPa}$ before increasing to higher values of $\sim 1-2 \mathrm{nPa}$ for approximately two days before decreasing again to low values. This interval is suitable for case studies of the influence of the dynamic pressure for several reasons. There are simultaneous measurements from MEX and MGS with all instruments running while crossings of the MPB and the BS by MEX occur at different solar zenith angles (SZAs) which give an indication of the shape of the boundaries. There are also times when MGS is at low SZAs and has a chance of seeing the MPB being pushed inward at the same time as MEX crosses the boundaries at other SZAs, which gives further indications of the shape of the boundaries. Furthermore, there are varying solar wind conditions during this interval so that we can observe how the boundaries respond to the different solar wind conditions while the EUV flux is steady. No boundary crossings occur over the strongest crustal fields where they can be significantly pushed upward. The BS and MPB crossings by MEX occur close to the plane which contains the IMF so that the convection electric field $\mathbf{E}_{\text {conv }}=-\mathbf{v} \times \mathbf{B}$ should have a small effect on the boundary location.

Figure 2 shows a seven day time series of data from MEX measurements of the solar wind dynamic pressure, MGS proxies for the dynamic pressure and IMF direction as well as the proxy for solar EUV flux. The general agreement between the pressure measured by MEX/IMA, in panel (a), and the pressure proxy, converted to magnetic pressure from MGS, in panel (b), is very good, with a correlation coefficient of 0.8 if the MGS values are interpolated to the time of the MEX values. We note that the MGS pressure proxy values during the interval in Fig. 2 are generally higher than the measured values from MEX/IMA. The three intervals on 3 July, 4 July and 5 July 2004 indicated by vertical black lines in Fig. 2 have been studied in more detail in Figs. 3-5, respectively.

Figure 3 shows a time series of MGS and MEX measurements and orbit geometry from the interval indicated as Case 1 on 3 July 2004 in Fig. 2. During this interval the solar 

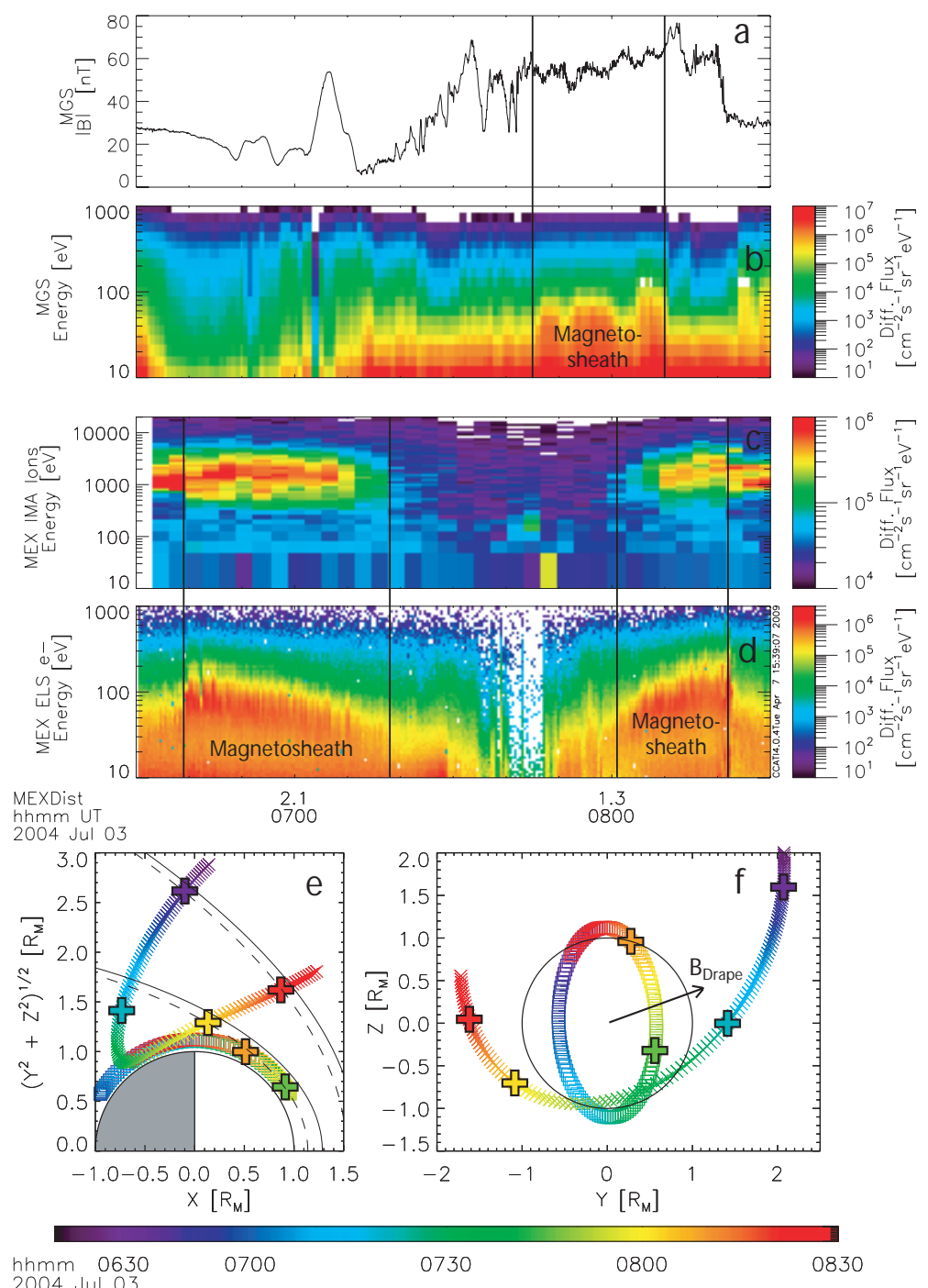

Fig. 3. MGS and MEX data for Case 1: time series of (a) MGS/MAG magnetic field strength, (b) MGS/ER omni-directional electron spectrogram, (c) MEX/IMA omni-directional ion spectrogram, (d) MEX/ELS omni-directional electron spectrogram and the orbits of MGS (squares) and MEX (crosses) in (e) cylindrical MSO coordinates and in (f) the MSO y-z-plane, colour coded by time. The black vertical lines in panels (a-d) indicate when MGS or MEX crosses the BS and MPB. The positions of those crossings are shown as large plus signs in panels (e) and (f). The black solid lines in panel (e) show the average location of BS and MPB (Vignes et al., 2000) and the black arrow indicate the direction of the magnetic field draping from MGS measurements. The black dashed lines indicate possible shapes of the BS and MPB based on the observed crossings in this case.

wind dynamic pressure is higher than normal as indicated by both MEX and MGS measurements in Fig. 2. MEX crosses the BS and MPB inbound at 06:39 UT and 07:18 UT, respectively, and outbound at 08:22 UT and 08:01 UT, respectively. The position of both boundaries is farther in than average. In between the inbound MPB crossing and the outbound BS crossing, from 07:45 UT until 08:10 UT, MGS observes magnetosheath electrons indicating that the MPB has moved to altitudes below $400 \mathrm{~km}$. The two dashed lines in panel (e) show possible shapes of the boundaries based on the two BS crossings by MEX and the three MPB crossings by MGS and
MEX that happen closest together in time. We have not taken into account the position of the MEX inbound MPB crossing since we assume that the boundaries move on a time scale less than $20 \mathrm{~min}$ and the inbound MPB crossing by MEX occurs 27 min earlier than the next crossing. Since we only have two BS crossings, we are left with including both of them. Obviously, we cannot produce statistical fits based on three or four crossings. The dashed lines have therefore been produced simply by manually adjusting conic sections so that they fit with the crossings of the MPB and BS and have similar shapes to the statistical best fits. They are hence only to 

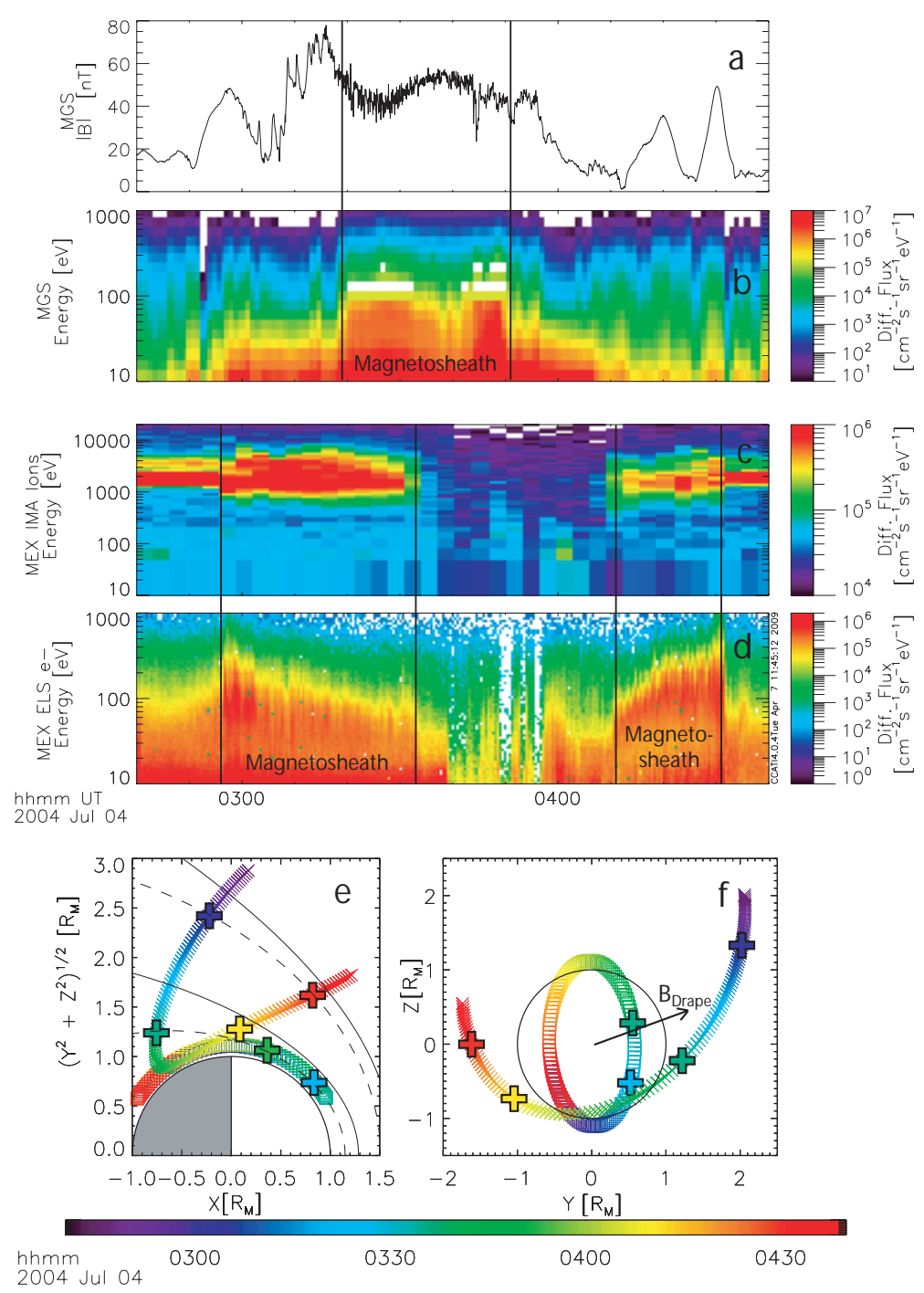

Fig. 4. As for Fig. 3 except for Case 2.

be taken as indications of what the shape of the boundaries might look like at this time.

Figure 4 shows data from the Case 2 interval on 4 July 2004 in Fig. 2 in the same format as Fig. 3. During this interval the pressure drops drastically from high $(\sim 2 \mathrm{nPa})$ to low $(\sim 1 \mathrm{nPa})$ values as shown in Fig. 2 . The dynamic pressure during the inbound leg of MEX is higher than in Case 1, such that the BS crossing observed by MEX at 02:56 UT and the subsequent MPB crossing at 03:33 UT consequently also occur closer in than in Case 1. The outbound MPB crossing at 04:11 UT and BS crossing at 04:31 UT are, however, located only slightly closer in compared to the outbound crossings in Case 1. The pressure during the outbound leg as observed by MEX had in fact dropped to only slightly higher values compared to the values from the outbound leg in Case 1. MGS also observed magnetosheath electrons during this interval, starting at 03:19 UT and ending at 03:51 UT. These magnetosheath observations started at roughly the same position in the $y-z$ plane as in Case 1 (compare panel (f) in Figs. 4 and 3 ) but stop much earlier in this case, at a lower $z$-value, probably due to the fact that the upstream pressure decreased and the magnetosheath moves outward again.

Figure 5 shows data from the Case 3 interval on 5 July 2004 in Fig. 2 in the same format as Figs. 3 and 4. At this time the pressure of the solar wind had decreased to lower values of $\sim 0.2 \mathrm{nPa}$ as shown both by the MGS pressure proxy and the MEX measurements in Fig. 2. The inbound BS crossing and MPB crossing observed by MEX at 05:45 UT and 06:15 UT, respectively, are still located farther in than average but have moved out compared to Case 2. The inbound BS crossing actually still occurs slightly farther in compared to the inbound BS crossings in Case 1, which indicates that 

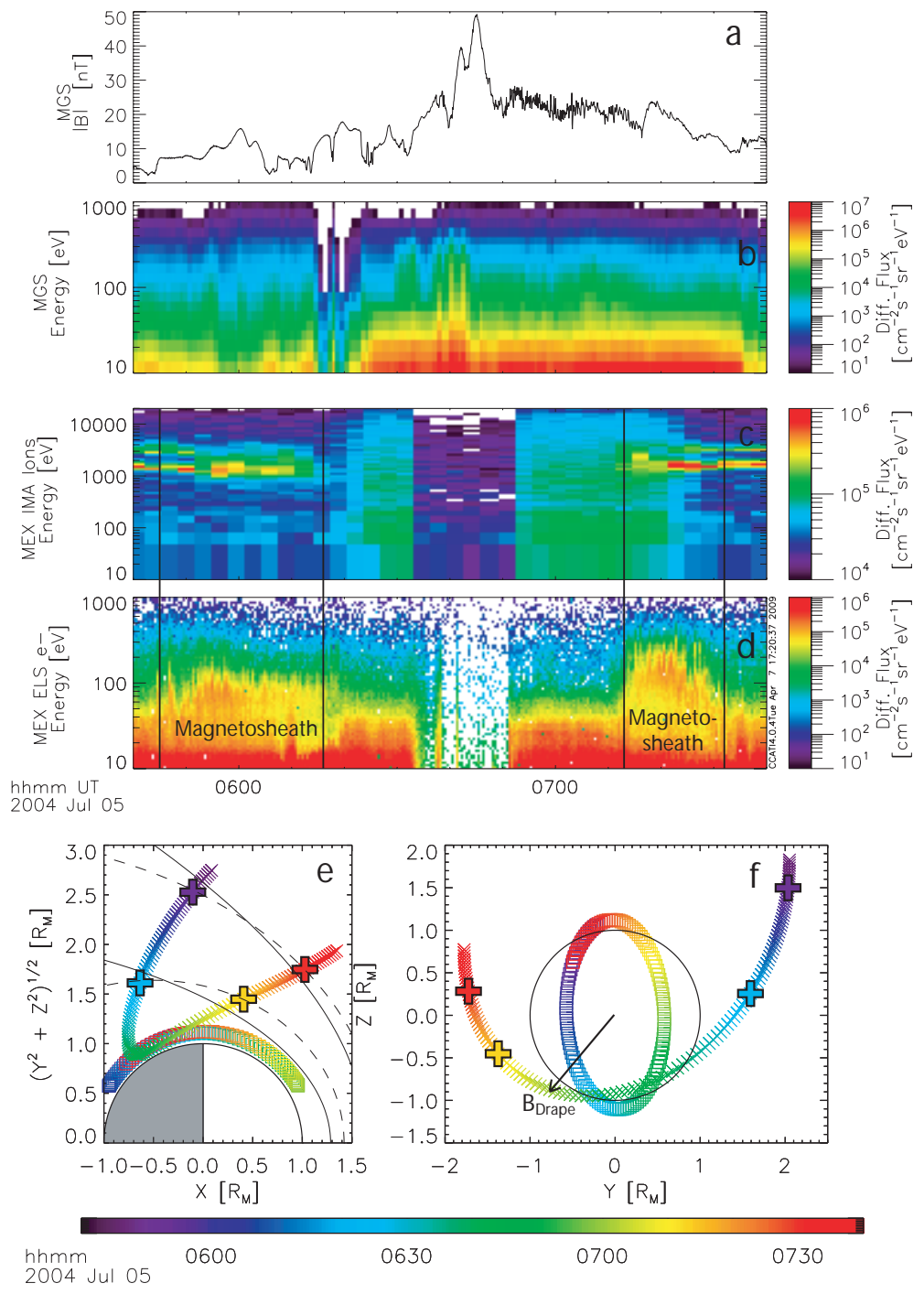

Fig. 5. As for Fig. 3 except for Case 3.

factors other than solar wind dynamic pressure are involved in determining the position of the boundary such as magnetosheath plasma pressure, magnetosonic Mach number, crustal fields (possibly on a more global scale since neither of the crossings occur directly over strong crustal fields) or EUV flux. The outbound MPB crossing at 07:13 UT and BS crossing at 07:32 UT have moved outward significantly and are now located at larger radius than their average positions. The outbound MPB and BS crossings by MEX also occur in the $-\mathbf{E}_{\text {conv }}$-hemisphere and are still farther out than average, indicating that the convective electric field has a weak influence on the boundary locations in this case. MGS observes no magnetosheath plasma at all during this interval. Evidently, the pressure was simply not high enough to push the MPB and the magnetosheath down to the altitude of MGS.
To summarize, both the MGS pressure proxy and MEX measurements indicate that the solar wind undergoes some changes in dynamic pressure during the above mentioned interval and this is found to be in agreement with the observed response of the plasma boundaries. The boundaries are pushed inward when the dynamic pressure is high, as shown in Fig. 3. When the dynamic pressure is even higher, as during the inbound part in Fig. 4, the boundaries are even farther in. The magnetosheath is in fact pushed inward to below the $400 \mathrm{~km}$ altitude of MGS. But when the pressure drops, which happens sometimes between 03:51 UT and 04:11 UT in Fig. 4, the boundaries respond and move outward quickly and we can conclude that the response time of the plasma environment of Mars to new solar wind conditions is at least less than $20 \mathrm{~min}$. From Fig. 5, we conclude that the boundaries can either take asymmetric forms 

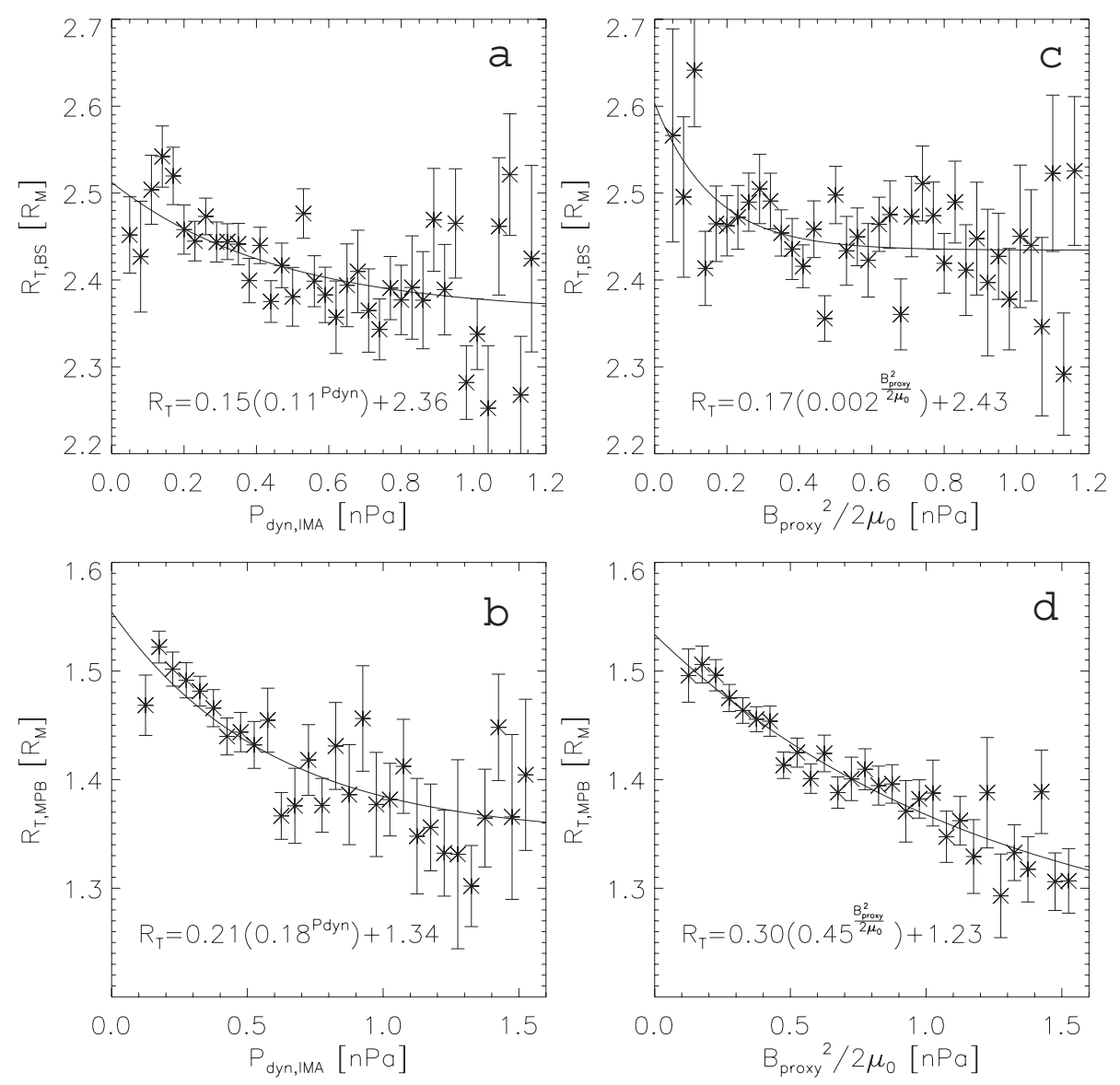

Fig. 6. The extrapolated terminator distance of all (a) BS and (b) MPB crossings observed by MEX from February 2004 until November 2006 plotted as a function of solar wind dynamic pressure measured outside of the BS by MEX/IMA and the extrapolated terminator distance of all (c) BS and (d) MPB crossings as a function of the MGS pressure proxy linearly interpolated to the time of the crossings. The curve in each panel is a least square exponential fit to the data. The error bars show standard error on the mean.

since they, during steady solar wind conditions, are farther out over one hemisphere than the other, or, that the shape of the boundaries does not follow the average best fit conic section shape in this case. The "asymmetry" does not seem to depend on the direction of the convective electric field, which could be a factor that causes asymmetry. This leads us to believe the latter of the two explanations.

\section{Statistical studies with simultaneous measurements}

\subsection{Influence of the solar wind dynamic pressure}

In Fig. 6 we show the result of a statistical study of how the radial distance of the BS and the MPB vary with solar wind dynamic pressure. We have used all the crossings observed by MEX during the overlapping mission time with MGS and extrapolated them to the terminator plane in order to remove the SZA dependence. The boundaries tend to be at higher altitudes at higher SZAs and in order to compare crossings with each other we need to remove this dependence and we therefore extrapolate the positions of all the crossings to the terminator plane. The extrapolation is done using the same method as described in Vignes et al. (2002) and Crider et al. (2002). First, the crossings are rotated by $4^{\circ}$ about the MSO $\mathrm{z}$-axis to account for the perpendicular movement of Mars relative to the solar wind flow direction. A conic section is then fitted to each crossing of the BS and MPB in the MSO $\left(x, \sqrt{y^{2}+z^{2}}\right)$ plane by using the best fit values of the eccentricity $\epsilon$ and $X_{0}$ from Edberg et al. (2008), varying only the semi-latus rectum $L$. For each conic section the terminator distance is then calculated. This method is not perfect, since the eccentricity of the boundaries can possibly also change when the governing factors change, but it remains the best we can do for now in order to remove the SZA dependence. Fitting curves by varying only $\epsilon$ or only $X_{0}$ does not give reasonable curves. Crossings that occur either much closer in than average or far downtail tend to produce fitted curves that pass through the planet. Varying $L$ and keeping the other 
parameters fixed gives more reasonable curves in this sense. We therefore assume $\epsilon$ and $X_{0}$ to be constant.

Figure 6, panel (a), shows the extrapolated terminator distances $R_{T}$ of the BS crossings as a function of upstream solar wind dynamic pressure $P_{\text {dyn,IMA }}$ as measured by MEX/IMA. The crossings are binned into $0.05 R_{M}$ bins and the mean values of the dynamic pressure upstream of all crossings in each bin is calculated. The error bars show standard error on the mean (standard deviation divided by number of samples in each bin). There is a trend to smaller radial distance for higher dynamic pressure, with a correlation coefficient of -0.51 , and we fit an exponential curve (solid line) of the form $R_{T}=a b^{P_{\mathrm{dyn}}}+c$, where $a, b$ and $c$ are free parameters, to the data points. In panel (b) we show the terminator radius of the MPB as a function of $P_{\mathrm{dyn}, \mathrm{IMA}}$. Again, there is a clear trend to smaller radial distances for higher $P_{\text {dyn,IMA, }}$, with a correlation coefficient of -0.74 , and we fit the same type of exponential curve to the data points. The error bars increase in size with falling radial distance, which could be an effect of the stronger influence of the crustal fields at lower altitudes. However, it could also be an effect of fewer data points in these bins. These two results clearly show that the solar wind dynamic pressure has an influence on the location of the boundaries, as would be expected.

We compare these results to those obtained when we use the MGS pressure proxy rather than $P_{\text {dyn,IMA. Panels (c) and }}$ (d) show the terminator radius of the BS and MPB, respectively, as a function of the MGS pressure proxy $\frac{B_{\text {proxy }}^{2}}{2 \mu_{0}}$. The pressure proxy values are linearly interpolated to the time of the boundary crossings. Surprisingly, there is no obvious trend for the variation of the BS radius (correlation coefficient of -0.41) whereas the trend for the MPB is very similar to that in panel (b) (correlation coefficient of -0.93). The lack of a trend for the BS crossings could be explained by the time difference between the time of the pressure proxy measurement and the BS crossings, which can be as long as $1 \mathrm{~h}$. The BS is expected to move on time scales much shorter than that. The error bars are quite large, however, and including them, the results are not so discrepant. For the MPB we should have the same problem with the time difference but the results in panels (b) and (d) are still very similar, which rather disproves the argument above, if we assume that the BS and MPB move on the same timescales. It is also likely that the MPB and the BS can simply respond differently to changes in the solar wind dynamic pressure. The pressure proxy and the measured pressure values do not match up perfectly and this could be an indication of an unknown compression factor between the solar wind dynamic pressure outside the BS/MPB and the magnetic pressure inside the MPB.

It is also possible that when the BS is at very low radial distances the crustal magnetic fields become more important while at the same time the dynamic pressure becomes less important. The dynamic pressure can only push the bound-
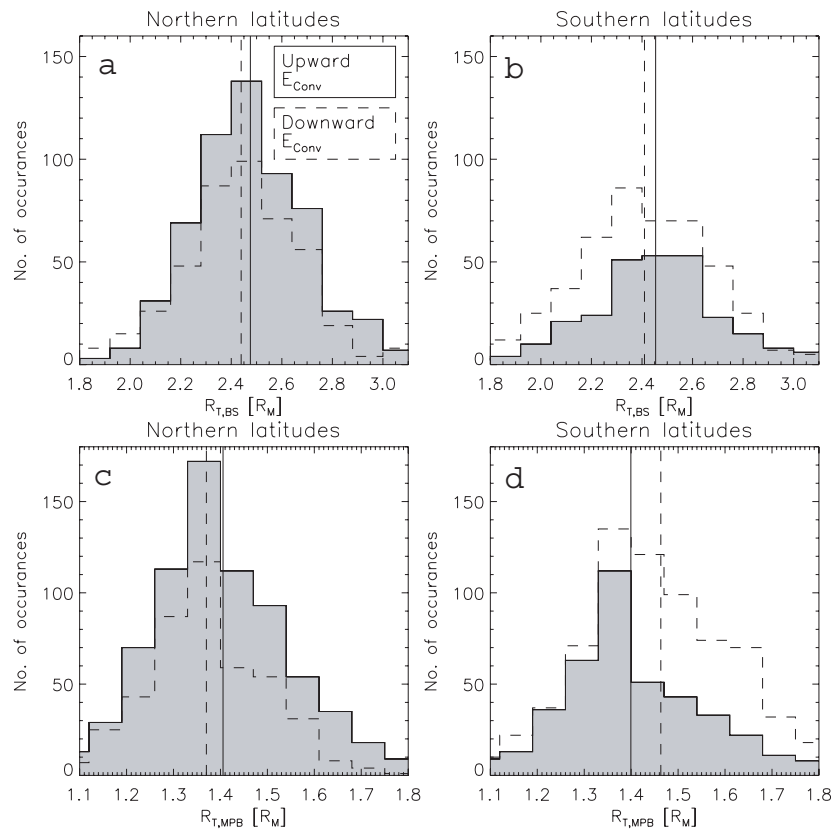

Fig. 7. Distribution of the extrapolated terminator distance of BS crossings in the (a) Northern and in the (b) Southern Hemisphere and MPB crossings in the (c) Northern and in the (d) Southern Hemisphere observed by MEX from February 2004 until November 2006. In each panel, the distributions are subdivided into whether they occur when the convective electric field is directed locally upward (solid, filled) or locally downward (dashed). The mean extrapolated terminator distances are shown for crossings that occur in the locally upward convective electric field hemisphere (solid vertical line) and in the locally downward convective electric field hemisphere (dashed vertical line).

ary down to a certain altitude before the magnetic pressure from the crustal fields together with the plasma pressure inside of the BS becomes too high and the trend of a lower radial distance for a higher dynamic pressure vanishes. Similarly, when the BS is at very high radial distances the IMF direction could become more important while at the same time the dynamic pressure becomes less important. Inclusion of crossings that occur when other parameters, such as IMF direction, solar EUV flux and crustal magnetic fields, are kept approximately constant would be a way to decrease the error bars and get clearer trends. Unfortunately, the number of data points in each bin drops drastically by doing those selections and the error bars increase rather than decrease. However, the trends are still similar. The same drastic decrease in number of data points happens if we try to match MEX crossings with MGS proxy values that occur closer together in time. We therefore choose to include all crossings when examining the influence of each factor. 

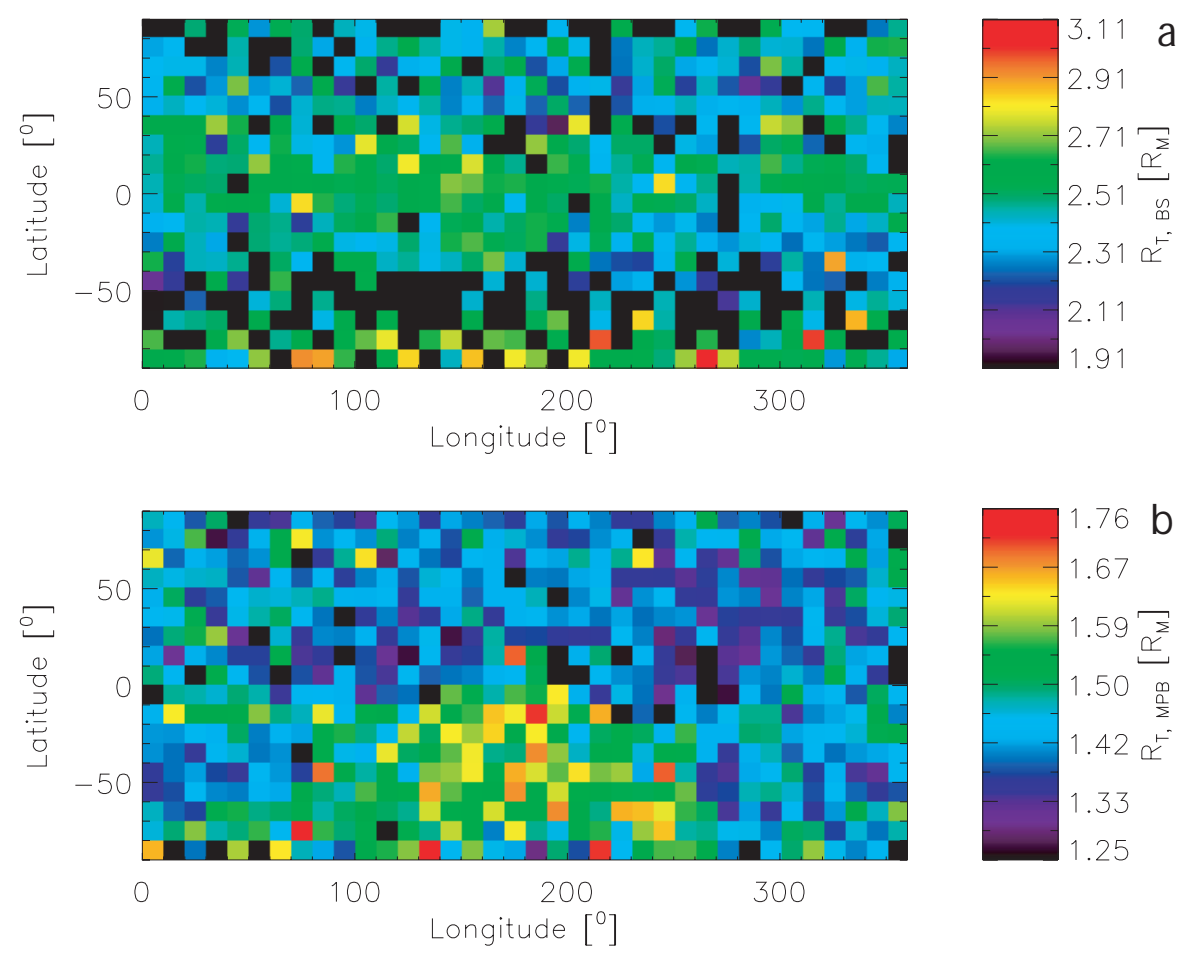

Fig. 8. Longitude-latitude maps colour coded by the extrapolated terminator distance $R_{T}$ of the (a) BS and (b) MPB as observed by MEX. Only dayside crossings were used. Black bins means that there were less than two crossings in that region.

\subsection{Influence of the IMF direction}

In Fig. 7 we show the result of a statistical study of the effect of the IMF direction on the boundary locations. The convective electric field $\mathbf{E}=-\mathbf{v} \times \mathbf{B}$ should cause the ions to go out into the solar wind and cause massloading of the solar wind on one side of the planet, creating a larger obstacle to the flow and thereby lifting the boundaries upward. The ions on the other side simply go back into the planet, as suggested by Vignes et al. (2002). In panels (a) and (b) the distribution of the radial distances of BS crossings at northern and southern latitudes of Mars, respectively, are shown, divided into whether the crossing occur during locally upward $(+E)$ or locally downward $(-E)$ convective electric field. The direction of the magnetic field is obtained from the MGS draping proxy. In panels (c) and (d) the distribution is shown for the MPB. The radial distance of the crossings are extrapolated to the terminator plane. In panel (a), the mean value of $R_{T}$ for the BS crossings that occur in the $+E$-hemisphere and at northern latitudes is $2.48 \pm 0.01 R_{M}$ (plus or minus standard error of the mean) and is $\sim 135 \mathrm{~km}$ higher compared to that in the $-E$-hemisphere at northern latitudes, $2.44 \pm 0.01 R_{M}$. The mean values are significantly different according to a Student's t-test at a 95\% confidence level. This difference is also observed for the crossings that occur at southern latitudes, in panel (b), and the difference is also significant according to a Student's t-test at a 95\% confidence level. The southern latitude mean distances are $2.45 \pm 0.01 R_{M}$ in the $+E$-hemisphere compared to $2.41 \pm 0.01 R_{M}$ in the $-E$ hemisphere, a difference of $\sim 135 \mathrm{~km}$.

For the MPB, Fig. 7, panel (c), there is also a significant (according to a Student's t-test at a 95\% confidence level) difference of $\sim 135 \mathrm{~km}$ between the mean values of the $+E$ and $-E$ hemisphere crossings at northern latitudes, $1.41 \pm 0.01 R_{M}$ compared to $1.37 \pm 0.01 R_{M}$. But for the crossings that occur at southern latitudes, in panel (d), the situation is reversed. The $+E$ hemisphere crossings have a $\sim 200 \mathrm{~km}$ lower mean value than the $-E$ hemisphere crossings, $1.40 \pm 0.01 R_{M}$ compared to $1.46 \pm 0.01 R_{M}$. The Southern Hemisphere MPB apparently reacts differently to an upward/downward convective electric field compared to the Northern Hemisphere, on average. It should be noted that the scatter in the extrapolated terminator distances is large and there are uncertainties involved in this study, e.g. the IMF direction is not determined perfectly, but only through a proxy and the time difference between the proxy value and the crossing has not been taken into account.

\section{Influence of the crustal magnetic fields}

In order to investigate the influence of the crustal magnetic fields we only use MEX measurements. The crustal fields 

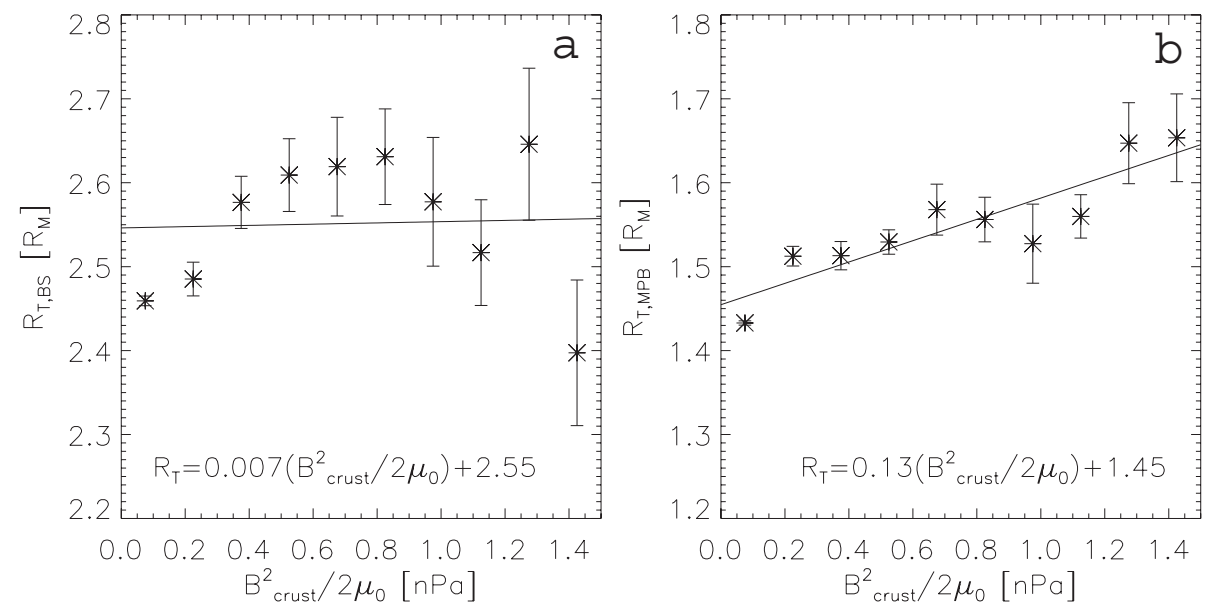

Fig. 9. Extrapolated terminator distance of all the (a) BS and (b) MPB crossings observed by MEX from February 2004 until January 2009 plotted as a function of the magnetic pressure from the crustal magnetic field as calculated from the model by Cain et al. (2003). The solid lines are fitted curves to the data points and the error bars show standard error on the mean values.

have previously been shown to influence the boundaries by using MGS measurements and also MEX measurements on their own (Crider et al., 2002; Brain et al., 2005; Fränz et al., 2006b; Edberg et al., 2008). However, no previous study has used such a large data set of crossings as we have in this study and we can now for the first time produce global maps of the radial distance of the boundaries. Panels (a) and (b) in Fig. 8 shows two global longitude-latitude maps colour coded by the radial distance, in $10^{\circ} \times 10^{\circ}$ bins, of the BS and MPB, respectively. All crossings from February 2004 until January 2009 from the dayside of Mars are used and the mean of the radial distance of all crossings within each bin are shown. Bins with less than two crossings are colored black. Note that the strongest crustal fields are located in the Southern Hemisphere at longitudes between $90^{\circ}$ and $270^{\circ}$ (Connerney et al., 2005). The map of the BS crossings, panel (a), shows no distinct influence of the crustal magnetic fields on the altitude of boundary, i.e. there is no specific region where the boundary is at larger radial distance than elsewhere and which also corresponds to a region of strong crustal fields. There are, unfortunately, many empty bins at southern latitudes where the crustal fields are strongest. However, the mean value of $R_{T}$ of all the dayside BS crossings in the Northern Hemisphere is $2.45 R_{M}$ compared to $2.49 R_{M}$ in the Southern Hemisphere and in the bottom rows of panel (a), at southern latitudes, there is a weak tendency that the crossings are at higher distances. The difference is statistically significant according to a Student's t-test at $95 \%$ confidence level. This difference is smaller than the difference presented in Edberg et al. (2008) where MGS crossings where used. The accuracy in this study should, however, be better due to the much larger data set.

The influence of the crustal magnetic fields on the MPB is much clearer. In panel (b) of Fig. 8 there is a large area at southern latitudes between longitudes from $\sim 90^{\circ}$ to $\sim 270^{\circ}$, where the MPB occurs at larger radial distances than elsewhere. This region corresponds very closely to the region where the strongest crustal fields are located. Also, at latitudes above $-30^{\circ}$ and at longitudes between $0^{\circ}$ and $90^{\circ}$ there is a less prominent but still visible area of higher MPB which corresponds to a region of intermediately strong crustal fields.

In Fig. 9 we show the extrapolated terminator distances of all the dayside crossings of the BS and MPB plotted as functions of magnetic pressure contributed from the crustal anomalies. The magnetic field strength is estimated by using the model from Cain et al. (2003) and calculating the field strength at 100 positions evenly spaced on a $10^{\circ} \times 10^{\circ}$ longitude-latitude grid centered radially below each crossing at $400 \mathrm{~km}$. The mean over these 100 values is then taken as the field strength for each individual crossings. We choose this method since the crustal field has a high spatial variability and just calculating the crustal field value at the exact location of the crossing will not necessarily give a correct estimate. The crossings are then binned in $0.15 \mathrm{nPa}$ bins and the mean value of the distance for all crossings within each bin is calculated. For the BS, we fit a linear least-square curve to the data points, as given by the equation in the figure. There is, as indicated before, a trend of a larger distance for higher crustal magnetic pressure than for low crustal field magnetic pressure but on the whole data set there is a very weak dependence. However, there are few crossings over strong crustal fields such that the uncertainty becomes larger for larger crustal magnetic field strength. For the MPB, the linear increase of the distance with increasing crustal magnetic pressure is more clear such that the crustal magnetic pressure has a strong influence on the MPB location. It should be mentioned that the scatter in position of crossings is extensive and the data presented are only mean values. 

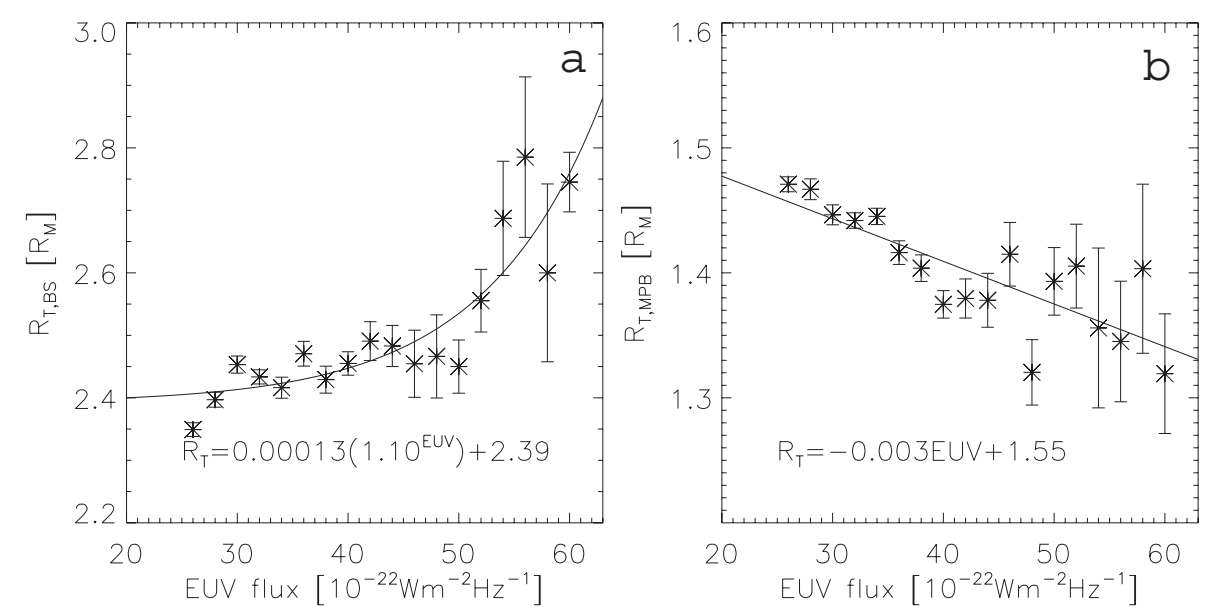

Fig. 10. Extrapolated terminator distance of the (a) BS and (b) MPB crossings observed by MEX from February 2004 until January 2009 plotted as a function of solar EUV flux proxy. The solid lines are fitted curves to the data points and the error bars show standard error on the mean values.

\section{Influence of the solar EUV flux}

In Fig. 10 we show how the the solar EUV flux affects the location of the BS and MPB. The crossings are divided into $2.0 \times 10^{-22} \mathrm{~W} \mathrm{~m}^{-2} \mathrm{~Hz}^{-1}$ bins and the mean of the radial distance for all crossings within each bin is calculated. There is a clear trend of a larger BS radius for a higher EUV flux and it seems to increase exponentially. For the BS we therefore fit an exponential curve of the form $R_{T}=a b^{P_{\mathrm{dyn}}}+c$, where $a$, $b$ and $c$ are free parameters as shown in panel (a). The MPB on the other hand clearly decreases in radius when the solar EUV flux increases and it seems to decrease linearly as the fit in panel (b) shows. Modolo et al. (2006) used hybrid simulations to study the influence of the EUV flux on the plasma boundary and found that the BS was pushed outward at the subsolar point but moved in at the terminator plane when going from solar minimum to maximum conditions, while the MPB only moved inward at the terminator, in agreement with this study. The data used in this study are all taken during the declining phase of the solar cycle and during solar minimum (2004-2009) and we can not yet determine how the EUV flux at solar maximum will affect the boundaries.

\section{Conclusions}

We have shown case studies and statistical studies using single as well as two-spacecraft simultaneous and nonsimultaneous measurements of how the solar wind dynamic pressure, the IMF direction, the crustal magnetic fields and the solar EUV flux affect the BS and MPB. We have produced expressions for how these factors influence the boundaries which enables us to determine their relative importance. We have not been able to study the influence of the solar wind magnetosonic Mach number since we do not have upstream magnetic field measurements. At Venus for instance, the magnetosonic Mach number has been shown to be of importance in determining the position of the BS (Russell et al., 1988) and it is expected that it should play a role at Mars too.

The case studies (Figs. 3-5) employ simultaneous MEX and MGS measurements during an interval when the solar wind dynamic pressure changes from high to low and the dynamic pressure is assumed to have the main influence on the boundary locations. The effect of the high pressure solar wind on the boundaries is indeed observed to be an inward movement of both boundaries. The MPB is in fact pushed closer to the planet than the altitude of MGS $(\sim 400 \mathrm{~km})$. Both the BS and the MPB remain compressed until the solar wind dynamic pressure has decreased back to lower values. From the case study, we can provide an upper limit of $\sim 20$ min for how long it takes for the Martian plasma environment to adapt to new upstream solar wind conditions (Fig. 4). However, a more detailed study is needed in the future to more accurately determine this time scale. The shapes of the boundaries seem to alter during this time as well as indicated by near-simultaneous measurements of the MPB by MEX and MGS (Fig. 4, panel f). The BS, which is only observed by MEX and separated in time from the inbound to the outbound by $\sim 1.5 \mathrm{~h}$, making it far from simultaneous, might also change its shape (Figs. 4 and 5, panel f). However, the BS is expected to move on time scales much shorter than this and this shape estimate is therefore very uncertain.

A statistical study of how the position of all MEX boundary crossings during the time of the mission overlap with MGS (February 2004-November 2006) varies with solar wind dynamic pressure has also been performed (Fig. 6). The boundaries are, on average, found to decrease exponentially in altitude with increasing pressure. Very similar result are 
produced when using the solar wind dynamic pressure as determined from a proxy from MGS data and by direct measurements from MEX. The statistical studies in Fig. 6 also provide confidence in the accuracy of the MGS and MEX measurement, which independently give very similar result. However, for the BS the method of using the MEX measurements give a seemingly clearer result to how the radial distance decreases with increasing dynamic pressure. If the MGS pressure is used, the trend is less clear but still visible. The BS could possibly move on shorter time scales than the MPB and the MGS pressure proxy has a too poor time resolution which would mean that the MGS proxy is not suitable for studying the variation of MEX BS crossings with dynamic pressure. Also, the trend for the MPB appears clearer if the MGS pressure proxy is used rather than if the MEX/IMA measurements are used. These results indicate that the BS and MPB do not respond in exactly the same way when facing changes in the upstream solar wind conditions.

A statistical study of how the boundaries react to different direction of convective electric field has also been performed by using two-spacecraft measurements (Fig. 7). We show that the BS has a $135 \mathrm{~km}$ larger average radial distance in the hemisphere of locally upward convective electric field compared to that in the hemisphere of locally downward convective electric field. The same difference is valid for the MPB but only in the Northern Hemisphere where the boundary during locally upward convective electric field has a $135 \mathrm{~km}$ larger average radial distance compared to when locally downward. In the southern latitudes, the situation seems to be reversed and the MPB is closer to the planet in the hemisphere of locally upward electric field, by $200 \mathrm{~km}$ on average. The difference is possibly due to the influence of the crustal magnetic fields, which are strongest in the Southern Hemisphere and disturb the (weak) effect of asymmetric massloading. Overall, the IMF direction has a weak influence on the boundaries if compared to other factors.

We also confirm observations, by using MEX measurements only, that the crustal fields affect the MPB strongly but the BS to a lesser extent by providing additional magnetic pressure. The radial distance of the MPB increases linearly with the crustal magnetic pressure.

The EUV flux, which ionises neutrals in the extended exosphere of Mars and increases the plasma pressure, is also shown to have a strong influence on both boundaries. The BS radius increases exponentially with increasing EUV flux while the MPB radius is observed to decrease linearly with increasing EUV flux. An explanation for this could be that the plasma pressure in the magnetosheath, in between the BS and the MPB, could be significantly increased with increasing ionisation from EUV flux which could cause the BS to move outward and the MPB to move inward. We have not found a correlation between solar EUV flux and high dynamic pressure which could have explained the result for the MPB.
The are several sources of error involved in this study, which causes scatter in the location of the boundaries. These errors include the time delay between MGS proxy values/upstream MEX measurements and the boundary crossings, the unknown $\mathrm{X}$-component of the magnetic field and the unknown upstream magnetosonic Mach number. A sheared solar wind flow would also distort the results. The solar EUV proxy could introduce an error since it is extrapolated from Earth and so can the extrapolation of the boundary distances to the terminator plane do.

In summary, quantitative expressions for the main factors the affect the BS and the MPB have been produced. The factors that mainly affect the MPB include the solar wind dynamic pressure, crustal magnetic fields and solar EUV flux while the IMF direction has a weaker influence. For the BS, the solar wind dynamic pressure and the solar EUV flux are the main controlling factors while the IMF direction and crustal magnetic fields play a minor role.

Acknowledgements. NJTE was supported by the European Union 6th Framework, contract MEST-CT-2004-7512. ML and SWHC were supported by STFC grant PP/E000983/1. We wish to thank the MGS MAG/ER team and the MEX ASPERA-3 team for providing the data used in this study.

Topical Editor R. Nakamura thanks R. Lundin and another anonymous referee for their help in evaluating this paper.

\section{References}

Acuña, M. H., Connerney, J. E. P., Wasilewski, P., Lin, R. P., Anderson, K. A., Carlson, C. W., McFadden, J., Curtis, D. W., Mitchell, D., Reme, H., Mazelle, C., Sauvaud, J. A., D’Uston, C., Cros, A., Medale, J. L., Bauer, S. J., Cloutier, P., Mayhew, M., Winterhalter, D., and Ness, N. F.: Magnetic field and plasma observations at Mars: Initial results of the Mars Global Surveyor mission, Science, 279, 1676-1680, 1998.

Barabash, S., Lundin, R., Andersson, H., Brinkfeldt, K., Grigoriev, A., Gunell, H., Holmström, M., Yamauchi, M., Asamura, K., Bochsler, P., Wurz, P., Cerulli-Irelli, R., Mura, A., Milillo, A., Maggi, M., Orsini, S., Coates, A. J., Linder, D. R., Kataria, D. O., Curtis, C. C., Hsieh, K. C., Sandel, B. R., Frahm, R. A., Sharber, J. R., Winningham, J. D., Grande, M., Kallio, E., Koskinen, H., Riihelä, P., Schmidt, W., Säles, T., Kozyra, J. U., Krupp, N., Woch, J., Livi, S., Luhmann, J. G., McKenna-Lawlor, S., Roelof, E. C., Williams, D. J., Sauvaud, J.-A., Fedorov, A., and Thocaven, J.-J.: The Analyzer of Space Plasmas and Energetic Atoms (ASPERA-3) for the Mars Express mission, Space Sci. Rev., 126, 113-164, doi:10.1007/s11214-006-9124-8, 2006.

Brain, D. A., Halekas, J. S., Lillis, R., Mitchell, D. L., Lin, R. P., and Crider, D. H.: Variability of the altitude of the Martian sheath, Geophys. Res. Lett., 32, L18203, doi:10.1029/2005GL023126, 2005.

Cain, J. C., Ferguson, B. B., and Mozzoni, D.: An n = 90 internal potential function of the Martian crustal magnetic field, J. Geophys. Res., 108(E2), 5008, doi:10.1029/2000JE001487, 2003.

Connerney, J. E. P., Acuña, M. H., Ness, N. F., Kletetschka, G., Mitchell, D. L., Lin, R. P., and Reme, H.: Tectonic implications 
of Mars crustal magnetism, Proc. Natl. Acad. Sci., 102, 1497014975, doi:10.1073/pnas.0507469102, 2005.

Crider, D. H., Acuña, M. H., Connerney, J. E. P., Vignes, D., Ness, N. F., Krymskii, A. M., Breus, T. K., Rème, H., Mazelle, C., Mitchell, D. L., Lin, R. P., Cloutier, P. A., and Winterhalter, D.: Observations of the latitude dependence of the location of the martian magnetic pileup boundary, Geophys. Res. Lett., 29, L8, doi:10.1029/2001GL013860, 2002.

Crider, D. H., Vignes, D., Krymskii, A. M., Breus, T. K., Ness, N. F., Mitchell, D. L., Slavin, J. A., and Acuña, M. H.: A proxy for determining solar wind dynamic pressure at Mars using Mars Global Surveyor data, J. Geophys. Res., 108(A2), 1461, doi:10. 1029/2003JA009875, 2003.

Dubinin, E., Fränz, M., Woch, J., Roussos, E., Barabash, S., Lundin, R., Winningham, J. D., Frahm, R. A., and Acuña, M.: Plasma morphology at Mars: Aspera-3 observations, Space Sci. Rev., 126, 209-238, doi:10.1007/s11214-006-9039-4, 2006.

Dubinin, E., Modolo, R., Fränz, M., Woch, J., Akalin, F., Gurnett, D., Lundin, R., Barabash, S., Plaut, J. J., and Picardi, G.: Structure and dynamics of the solar wind/ionosphere interface on Mars. MEX-ASPERA-3 and MEX-MARSIS observations, Geophys. Res. Lett., 35, L11103, doi:10.1029/2008GL033730, 2008.

Edberg, N. J. T., Lester, M., Cowley, S. W. H., and Eriksson, A. I.: Statistical analysis of the location of the Martian magnetic pileup boundary and bow shock and the influence of crustal magnetic fields, J. Geophys. Res., 113, A08206, doi:10.1029/ 2008JA013096, 2008.

Fedorov, A., Budnik, E., Sauvaud, J.-A., Mazelle, C., Barabash, S., Lundin, R., Acuña, M., Holmström, M., Grigoriev, A., Yamauchi, M., Andersson, H., Thocaven, J.-J., Winningham, D., Frahm, R., Sharber, J. R., Scherrer, J., Coates, A. J., Linder, D. R., Kataria, D. O., Kallio, E., Koskinen, H., Säles, T., Riihelä, P., Schmidt, W., Kozyra, J., Luhmann, J., Roelof, E., Williams, D., Livi, S., Curtis, C. C., Hsieh, K. C., Sandel, B. R., Grande, M., Carter, M., McKenna-Lawler, S., Orsini, S., Cerulli-Irelli, R., Maggi, M., Wurz, P., Bochsler, P., Krupp, N., Woch, J., Fränz, M., Asamura, K., and Dierker, C.: Structure of the Martian wake, Icarus, 182, 329-336, doi:10.1016/j.icarus.2005.09.021, 2006.

Fränz, M., Dubinin, E., Roussos, E., Woch, J., Winningham, J. D., Frahm, R., Coates, A. J., Fedorov, A., Barabash, S., and Lundin, R.: Plasma moments in the environment of Mars, Space Sci. Rev., 126, 165-207, doi:10.1007/s11214-006-9115-9, 2006a.

Fränz, M., Winningham, J. D., Dubinin, E., Roussos, E., Woch, J., Barabash, S., Lundin, R., Holmström, M., Andersson, H., Yamauchi, M., Grigoriev, A., Frahm, R. A., Sharber, J. R., Scherrer, J. R., Coates, A. J., Soobiah, Y., Linder, D. R., Kataria, D. O., Kallio, E., Säles, T., Riihelä, P., Schmidt, W., Koskinen, H. E. J., Kozyra, J., Luhmann, J., Roelof, E., Williams, D., Livi, S., Curtis, C. C., Hsieh, K. C., Sandel, B. R., Grande, M., Carter, M., Sauvaud, J.-A., Fedorov, A., Thocaven, J.-J., McKenna-Lawler, S., Orsini, S., Cerulli-Irelli, R., Maggi, M., Wurz, P., Bochsler, P., Krupp, N., Asamura, K., and Dierker, C.: Plasma intrusion above Mars crustal fields - Mars Express ASPERA-3 observations, Icarus, 182, 406-412, doi:10.1016/j.icarus.2005.11.016, 2006b.
Mitchell, D. L., Lin, R. P., Mazelle, C., Rème, H., Cloutier, P. A., Connerney, J. E. P., Acuña, M. H., and Ness, N. F.: Probing Mars' crustal magnetic field and ionosphere with the MGS Electron Reflectometer, J. Geophys. Res., 106, E10, doi: 10.1029/2000JE001435, 2001.

Modolo, R., Chanteur, G. M., Dubinin, E., and Matthews, A. P.: Simulated solar wind plasma interaction with the Martian exosphere: influence of the solar EUV flux on the bow shock and the magnetic pile-up boundary, Ann. Geophys., 24, 3403-3410, 2006, http://www.ann-geophys.net/24/3403/2006/.

Nagy, A. F., Winterhalter, D., Sauer, K., Cravens, T. E., Brecht, S., Mazelle, C., Crider, D., Kallio, E., Zakharov, A., Dubinin, E., Verigin, M., Kotova, G., Axford, W. I., Bertucci, C., and Trotignon, J. G.: The plasma environment of Mars, Space Sci. Rev., 111, 33-114, doi:10.1023/B:SPAC.0000032718.47512.92, 2004.

Russell, C. T., Chou, E., Luhmann, J. G., Gazis, P., Brace, L. H., and Hoegy, W. R.: Solar and interplanetary control of the location of the Venus bow shock, J. Geophys. Res, 93, 5461-5469, doi: 10.1029/JA093iA06p05461, 1988.

Slavin, J. A. and Holzer, R. E.: Solar wind flow about the terrestrial planets. I - Modeling bow shock position and shape, J. Geophys. Res., 86, 11 401-11418, 1981.

Trotignon, J. G., Mazelle, C., Bertucci, C., and Acuña, M. H.: Martian shock and magnetic pile-up boundary positions and shapes determined from the Phobos 2 and Mars Global Surveyor data sets, Planet. Space Sci., 54, 357-369, doi:10.1016/j.pss.2006.01. 003, 2006.

Vignes, D., Mazelle, C., Reme, H., Acuña, M. H., Connerney, J. E. P., Lin, R. P., Mitchell, D. L., Cloutier, P., Crider, D. H., and Ness, N. F.: The solar wind interaction with Mars: locations and shapes of the bow shock and the magnetic pile-up boundary from the observations of the MAG/ER experiment onboard Mars Global Surveyor, Geophys. Res. Lett., 27, 49-52, doi:10.1029/1999GL010703, 2000.

Vignes, D., Acuña, M. H., Connerney, J. E. P., Crider, D. H., Rème, H., and Mazelle, C.: Factors controlling the location of the bow shock at Mars, Geophys. Res. Lett., 29(9), 1328, doi:10.1029/ 2001GL014513, 2002. 